\title{
Smartphone: a new device for teaching Physics
}

\section{J. A. Sans, F. J. Manjón, V. Cuenca-Gotor, M. H. Giménez-Valentín, I. Salinas, J. J. Barreiro, J. A. Monsoriu, and J. A. Gomez-Tejedor*}

\author{
Departamento de Física Aplicada, Escuela Técnica Superior de Ingeniería del Diseño, \\ Universitat Politècnica de València, Camí de Vera s/n, 46022 València (Spain), ${ }^{*}$ jogomez@fis.upv.es
}

\begin{abstract}
This paper reports on the use of smartphone's sensors to perform several experiments designed to teach fundamentals of Physics. We have adapted traditional physics laboratory sessions to the use of the different sensors that can be found in a typical smartphone, such as an accelerometer, and light and magnetic field sensors. The existence of a large repository of free AndroidTM and AppleTM applications which exploit the characteristics of these sensors facilitates the design of new experiments. A survey was done to the students in order to obtain feedback and to evaluate the success of the experience. The results of the survey showed a good acceptance of this method triggering their curiosity, with an average mark of 9 over 10. This project offers to the student a new way to think on smartphones as an attractive tool for possible application in experimental measurements and scientific demonstrations and not only as a socializing tool.
\end{abstract}

Keywords: Smartphone, Physics, Lab practice

\section{Introduction}

The use of portable devices in all teaching levels of Physics has increased significantly in the past decade. Sensors found in these new electronic devices can be used as new instruments and detectors in experimental measurements in the Physics laboratory. For instance, digital cameras, ${ }^{1}$ webcams, ${ }^{2}$ optical computer mice, ${ }^{3,4}$ Wii classic controller, ${ }^{5}$ Xbox Kinect sensor ${ }^{6}$ and other video game console controllers ${ }^{7}$ have been already exploited. In this respect, Wiimote and Xbox Kinetic sensor are the most outstanding controllers to be considered since they can be applied to several Physics experiments. ${ }^{8}$ The use of the Wiimote allows tracking several objects simultaneously by Bluetooth connection thanks to the use of three axis accelerometers which describe the trajectory of the objects in 3D. Additionally, the Xbox Kinect sensor includes the timeline parameter to 3D positioning. The main drawback of these devices is that they require specific software and are not widely available in the Physics laboratory. On the contrary, the use of mobile phones among young people is widely spread and the constant evolution of their technology, as well as the availability of free applications, makes them an attractive tool to perform experimental measurements and scientific demonstrations.

Recently, our group has worked in the use of the different smartphone's sensors on Physics experiments. For instance, we exploited the use of the smartphone's digital gyroscope to study a system of coupled oscillators ${ }^{9,10}$ and presented a similar study using the smartphone's ambient light sensor. ${ }^{11}$ Additionally, we used the smartphone's microphone to measure Doppler effect in sound waves. ${ }^{12,13}$ This background has allowed us to design and perform new experiments based on the smartphone's sensors previously described and even propose new experiments using the magnetic field sensor in order to explore the Physics of Electromagnetism. 


\section{Methods}

We have designed three different experiments in order to exploit the characteristics of the accelerometer, ambient light and magnetic field smartphone's sensors. The experiments are designed to cover different Physics fields leading from oscillatory movement, optics, and electromagnetics. All these experiments are based on classical lab practices, whose functioning have been tested along several years. The experiments proposed are:

\section{Experimental setup to characterize spring properties with the accelerometer sensor}

The use of the accelerometer sensor is suitable for experiments when the variation of the speed is decisive to characterize a certain physical magnitude. In this case, we propose the description of the oscillatory movement produced by a weight attached to a spring.

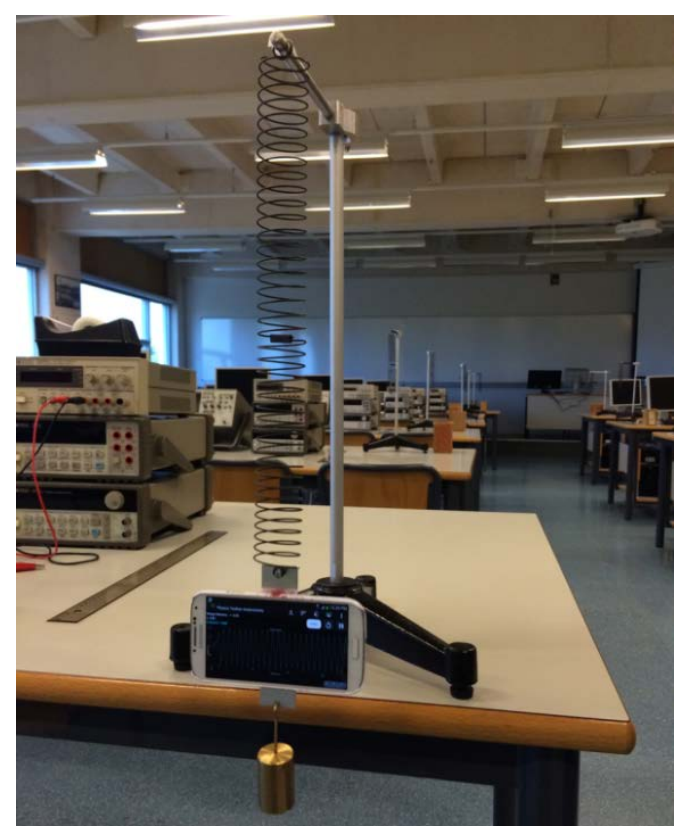

Figure 1. Picture of the experimental setup designed to measure the properties of the spring using the accelerometer sensor of the smartphone

The modification of the classical experimental setup consists in the introduction of a smartphone between the spring and the calibrated weight. The smartphone is glued with double-sided pressure-sensitive tape (or Scotch tape) to a thin aluminium sheet. The top extreme of the sheet is attached to the spring while on the bottom extreme of the sheet there is a hole for hanging the weights (see Fig. 1).

The oscillatory movement along the vertical axis $(z)$ of an object with mass $m$ hanged up to a spring with stiffness $k$ and shifted with respect to the equilibrium position is described by the simple harmonic movement equations:

$$
z(t)=A \sin \left(\omega_{0} t+\varphi\right)
$$




$$
T=\frac{2 \pi}{\omega_{0}}
$$

where $A$ is the shift amplitude, $\varphi$ is the initial phase and $\omega_{0}$ is the angular frequency, which is related to the oscillation period $T$ by Eq. (2). Once the frequency of the simple harmonic movement is known, the spring constant $k$ can be calculated using the following expression:

$$
k=m \omega_{0}^{2}
$$

The use of different calibrated weights allows obtaining an accurate value of the spring constant by a linear least-square fit of the square of the period of the oscillatory movement as a function of the corresponding weight of the system.

$$
T^{2}=\frac{4 \pi^{2}}{k} m
$$

\section{Experimental setup to measure the variation of the light intensity as a function of the distance to a light source using the ambient light sensor}

The smartphone's ambient light sensor allows adjusting the brightness of the screen to the environmental light in order to save battery and adequate the maximum visibility to the existent conditions. This ambient light sensor acts as a luxometer and there are several applications which allow determining in a quantitative way the light power measured by this sensor.

The experimental setup we propose for measuring the variation of the light intensity as a function of the distance to a light source is composed by a light source (dichroic halogen lamp without focusing optics) and a rule to measure the distance from the light source to the smartphone's ambient light sensor (see Fig. 2). With this simple setup it is possible to evidence the inverse proportionality between the light intensity and the square of the distance between the light source and the sensor. Care must be taken when using dichroic halogen bulbs since they cannot be touched with bare hands (neither before nor after its use). Note that, on one hand, bulbs can be poisoned when handled inappropriately, and, on the other hand, one can be burned if lamp is touched after its use because of the very high temperatures reached by the lamp filament. In this respect, we consider much better the use of high intensity light emitting diodes (LEDs) for the present experiment.

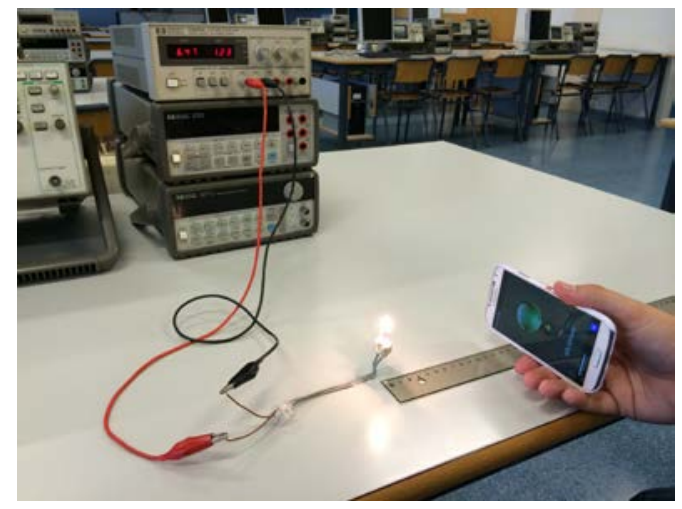

Figure 2. Picture of the experimental setup designed to measure the variation of the light intensity with the distance using the ambient light sensor of the smartphone. 
If we consider our lamp as a point light source, the radiation emitted by this one is spread by means of a spherical wave front. The ambient light sensor is considered as well as a point detector, due to their small area compared with the total spherical wave front. In this way, the intensity detected by the sensor is the light power of the lamp divided by the sphere area corresponding to the distance at which the sensor is placed. The light intensity measured by the smartphone at each distance will follow the inverse square law with the distance as follows:

$$
I(d) \propto I_{0} \frac{1}{d^{2}}
$$

\section{Experimental setup to measure the direction of the magnetic field induced by a DC electrical current in a linear wire}

Typically, smartphones contain three magnetic sensors perpendicular to each other. These sensors allow orienting the screen with respect to the Earth's magnetic field, which is very useful in navigation applications. We have used these sensors to describe the Oersted's experiment; i.e., to show how the direction of the magnetic field at the smartphone's location can change when the magnetic field induced by a continuous electrical current is added to the Earth's magnetic field. This experiment shows the relationship between electric and magnetic fields and was the origin of Electromagnetism.

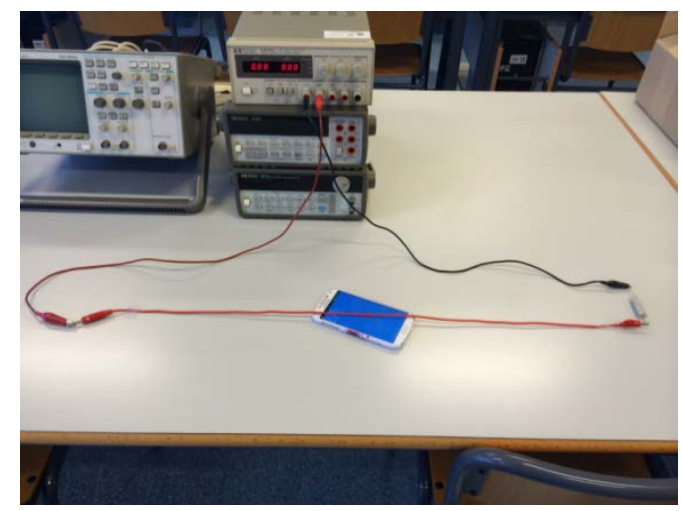

Figure 3. Picture of the experimental setup designed to measure the variation of the light intensity with the distance using the ambient light sensor of the smartphone.

In our setup, we plug a long linear wire and a serial resistance to a DC power supply. Then, we place the wire crossing over the smartphone's magnetic sensor in such a way that the wire is parallel to the direction of the Earth's magnetic field, as it is shown in Fig. 3. In this way, the direction of the magnetic field induced by the DC electrical current (obtained following the well-known right-hand rule) is perpendicular to the Earth's magnetic field and the deviation of the needle of the digital compass in the app (once the DC current is set on) is maximally visualized. 


\section{Results and Discussion}

\section{Periodic oscillatory movement}

We have used the free "Physics Toolbox Accelerometer" application to record the oscillatory movement exhibited by the system (smartphone + weights) as can be observed in Fig. 4(a). The curves plotted in the application allow determining the period (and frequency) of the system for different weights. Experimental data visualized in Fig. 4(a) show a weak damping that can be neglected as a first approximation. The period of the oscillation and the mass of the system are the parameters used to fit to Eq. (4), where a regression coefficient $\mathrm{R}^{2}$ higher that 0.995 was obtained in all cases, thus indicating the good quality of the data (see Fig. 4(b)).
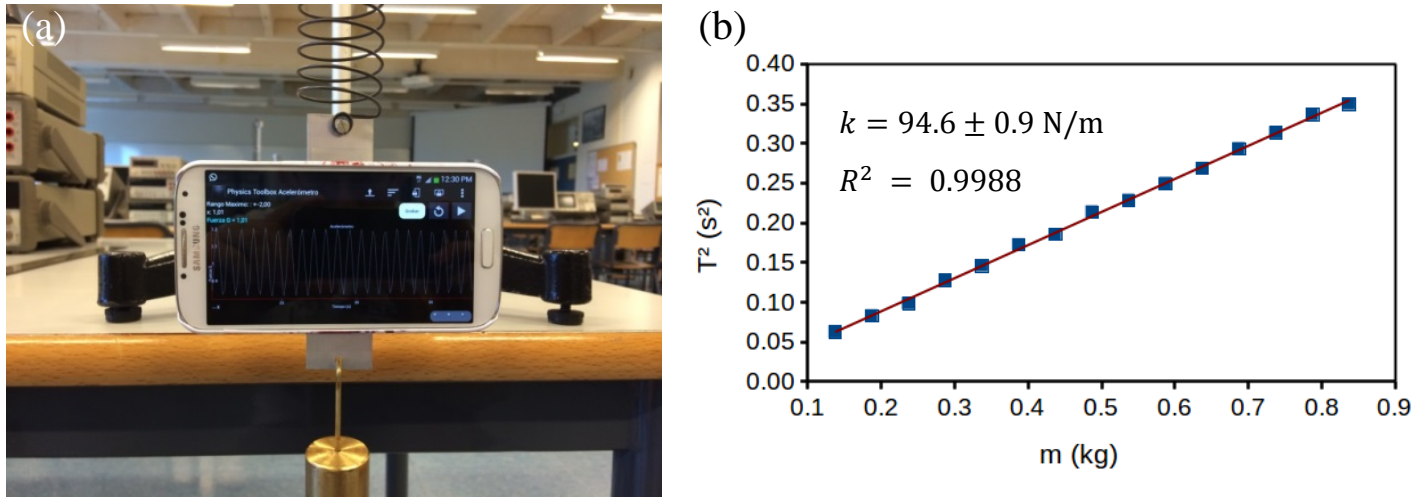

Figure 4. (a) Picture of the experimental curve obtained in the smartphone. (b) Fit of the experimental data to equation 4 to obtain a realistic value of the stiffness of the spring

\section{Inverse square law of light intensity vs distance}

We have used the "light sensor" section of the free application "Android sensor box". This application does not allow recording the intensity for each distance but we propose the students to plot the intensity value obtained as a function of the detector-source distance. The experimental intensity as a function of the inverse of the square of the detector-source distance will follow a linear trend line, as shown in Fig. 5(b).

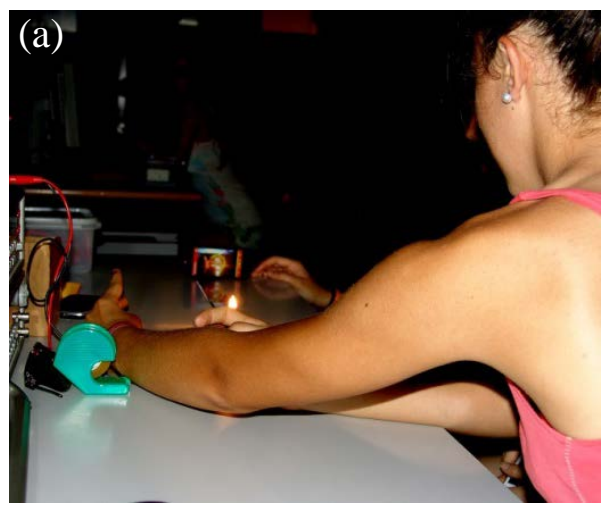

(b)

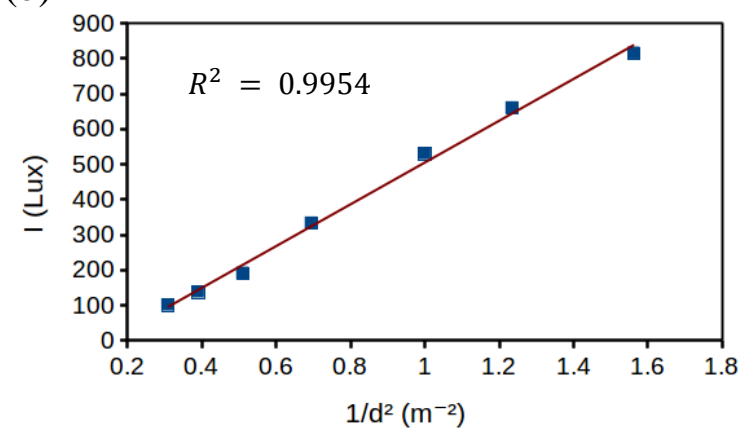

Figure 5. (a) Picture of the practice performed by students. (b) Experimental evolution of the intensity with inverse of the square of the detector-source distance. 


\section{Oersted's law experiment}

Unlike the previous two experiments proposed, the Oersted's law practice is only qualitative. Here, students are guided to identify the close relationship between electric and magnetic fields. The free application used to perform this experiment is "Magnetic Field Sensor". The introductory part of the practice serves to explain to the students how the magnetic field's lines turn around a linear wire.

First of all, students are told to find the position of the magnetic sensors in their smartphones. This could be done by rotating the speaker of a smartphone around another smartphone fixed with the application running or with the help of a small neodymium magnet. The position at which the magnetic flux is maximum determines the location of the sensor. Note that in case of using the neodymium magnet, magnet should not be placed too close to the smartphone in order to avoid interference of the strong magnetic field of the magnet with the inner processes of the own smartphone.

After knowing the position of the magnetic field sensor in each smartphone, students are triggered to place the linear wire crossing it, as shown in Fig. 3. At that moment, we encourage students to change the intensity and direction of the electrical current and study the variation in the orientation of the needle of the digital compass shown by the application.

\section{Report of the survey}

In order to obtain feedback from the students for improvement of the proposed lab experiences using smartphones, we designed a survey to elucidate the impact of these lab sessions on students. In general, we can appreciate a very good acceptance of the lab sessions by students with an average assessment of the sessions of 9 points over 10 (see Table 1). This result corresponds to the survey done to 30 students.

Table 1. Survey results obtained from students

\begin{tabular}{|l|c|}
\hline Application field of the question & $\begin{array}{c}\text { Average } \\
\text { (Mark over 10) }\end{array}$ \\
\hline 1: Teachers seemed to dominate the session's subject. & $\mathbf{9 . 7 5}$ \\
\hline 2: Teachers have solved every question with accuracy and clarity. & $\mathbf{8 . 2 1}$ \\
\hline 3: Teachers encouraged me to participate in the session. & $\mathbf{8 . 2 9}$ \\
\hline 4: Teachers achieved to stir my interest for the session's subject. & $\mathbf{7 . 3 8}$ \\
\hline 5: Session's objectives have been clearly exposed at the beginning. & $\mathbf{8 . 7 4}$ \\
\hline 6: Session's contents fit the general subject of the global project. & $\mathbf{6 . 7 5}$ \\
\hline 7: Duration of the session was suitable to the experiments proposed. & $\mathbf{8 . 3 8}$ \\
\hline 8: Methodology employed and activities were adequate. & $\mathbf{9 . 1 7}$ \\
\hline 9: Material used was adequate. & $\mathbf{9 . 4 6}$ \\
\hline 10: My average evaluation of the session is good. & $\mathbf{9 . 0 0}$ \\
\hline
\end{tabular}




\section{Conclusions}

The different sensors available in smartphones are useful tools for possible applications in experimental measurements and scientific demonstrations as we have proved along this article. The lab practices here designed cover different Physics areas and open the door to students to carry out their own experiments at home by exploiting the technology implemented in their smartphones. Here, we have presented the development of classical Fundamental Physics Lab practices using three different sensors of the smartphone. These practices were tested by students who passed a survey in order to check the validity of the experiences. The results of the survey show the success in the objectives proposed and the warm welcome of the experiments designed.

\section{Acknowledgements}

Authors would like to thank the Institute of Education Sciences of the Universitat Politècnica de València (Spain) for support of the Teaching Innovation Groups MoMa and e-MACAFI and for the financial support through PIME Project PIME/2014/A/031/B. J.A. Sans thanks “Juan de la Cierva” fellowship program for financial support and Instituto de Diseño para la Fabricación y Producción Automatizada de la Universitat Politècnica de València (Spain) for let him to get a venia docendi at the Departamento de Física de la Universitat Politècnica de València (Spain).

\section{References}

Monsoriu, J. A., Giménez, M. H., Riera, J., \& Vidaurre, A. (2005). Measuring coupled oscillations using an automated video analysis technique based on image recognition. European Journal of Physics, 26, 1149-1155.

Shamim, S., Zia, W., \& Anwar, M. S. (2010). Investigating viscous damping using a webcam. American Journal of Physics 78, 433-436.

Romulo, O. O. \& Franklin, K. N. (1997). The computer mouse as a data acquisition interface: Application to harmonic oscillators. American Journal of Physics 65, 1115-1118.

Ng, T. W. \& Ang, K. T. (2005). The optical mouse for harmonic oscillator experimentation. American Journal of Physics 73, 793-795.

Tomarken, S. L., Simons, D. R., Helms, R. W., Johns, W. E., Schriver, K. E. \& Webster, M. S. (2012). Motion tracking in undergraduate physics laboratories with the Wii remote. American Journal of Physics 80, 351-354.

Ballester, J. \& Pheatt, Ch. (2013). Using the Xbox Kinect sensor for positional data acquisition. American Journal of Physics 81, 71-77.

Vannoni, M. \& Straulino, S. (2007). Low-cost accelerometers for physics experiments. European Journal of Physics 28, 781-787.

Skeffington, A. \& Scully, K. (2012). Simultaneous Tracking of Multiple Points Using a Wiimote. The Physics Teacher 50, 482-484.

Castro-Palacio, J. C., Velázquez-Abad, L., Giménez, F. \& Monsoriu, J. A. (2013). A quantitative analysis of coupled oscillations using mobile accelerometer sensors. European Journal of Physics 34, 737-744. 
Castro-Palacio, J. C., Velázquez-Abad, L., Serrano, M. H., \& Monsoriu, J. A. (2013). Using a mobile phone acceleration sensor in physics experiments on free and damped harmonic oscillations. American Journal of Physics 81, 472-475.

Sans, J. A., Manjón, F. J., Pereira, A. L. J., Gómez-Tejedor, J. A., \& Monsoriu, J. A. (2013). Oscillations studied with the smartphone ambient light sensor. European Journal of Physics 34, 1349-1354.

Gómez-Tejedor, J.A., Castro-Palacio, J.C., \& Monsoriu J.A. (2014). The acoustic Doppler effect applied to the study of linear motions. European Journal of Physics 35 (2), 25006-25015.

Gómez-Tejedor, J.A., Castro-Palacio, J.C., \& Monsoriu J.A. (2015). Frequency Analyser: A New Android Application for High Precision Frequency Measurement. Computer Applications in Engineering Education. DOI 10.1002/cae.21618 